\title{
Analytic self-gravitating 4-Baryons, traversable NUT-AdS wormholes, flat space-time multi-Skyrmions at finite volume and a novel transition in the $S U(3)$-Skyrme model
}

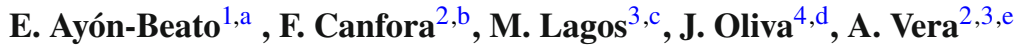 \\ ${ }^{1}$ Departamento de Física, CINVESTAV-IPN, Apdo. Postal 14-740, 07000 Mexico D.F., Mexico \\ ${ }^{2}$ Centro de Estudios Científicos (CECS), Casilla 1469, Valdivia, Chile \\ ${ }^{3}$ Instituto de Ciencias Físicas y Matemáticas, Universidad Austral de Chile, Valdivia, Chile \\ ${ }^{4}$ Departamento de Física, Universidad de Concepción, Casilla 160-C, Concepción, Chile
}

Received: 30 August 2019 / Accepted: 14 April 2020 / Published online: 8 May 2020

(c) The Author(s) 2020

\begin{abstract}
We construct the first analytic self-gravitating Skyrmions with higher Baryon charge in four dimensions for the $S U$ (3)-Skyrme-Einstein- $\Lambda$ theory by combining the generalized hedgehog ansatz with the approach developed by Balachandran et al. to describe the first (numerical) example of a non-embedded solution. These are genuine $S U(3)$ analytic solutions instead of trivial embeddings of $S U(2)$ into $S U$ (3) and its geometry is that of a Bianchi IX Universe. The Skyrme ansatz is chosen in such a way that the Skyrme field equations are identically satisfied in the sector with Baryon charge 4 . The field equations reduce to a dynamical system for the three Bianchi IX scale factors. Particular solutions are explicitly analyzed. Traversable wormholes with NUT-AdS asymptotics supported by a topologically non-trivial $S U$ (3)sigma soliton are also constructed. The self-gravitating solutions admit also a suitable flat limit giving rise to Skyrmions of charge 4 confined in a box of finite volume maintaining the integrability of the $S U$ (3) Skyrme field equations. This formalism discloses a novel transition at finite Baryon density arising from the competition between embedded and nonembedded solutions in which the non-embedded solutions prevail at high density while are suppressed at low densities.
\end{abstract}

\section{Introduction}

One of the most important results in low-energy quantum chromodynamics (QCD) is that its effective action becomes

\footnotetext{
a e-mail: ayon-beato@fis.cinvestav.mx

be-mail: canfora@cecs.cl

c e-mail: marcelagosf@gmail.com

de-mail: julioolivazapata@gmail.com (corresponding author)

e e-mail: avera@cecs.cl
}

the Skyrme model [1,2]; a Bosonic action for a $S U(N)$ valued scalar field [3-5], the physical case being $S U(3)$. Its solitons, dubbed Skyrmions, represent Fermionic states whose topological charge is the Baryon number $[1,2,6]$, see also [7-10]. These results have been also generalized to curved space-times $[11,12]$. The Skyrme model has been deeply analyzed not only by its emergence in low energy QCD but also due to its relevance in General Relativity. For instance, black holes with a non-trivial Skyrme hair were found using numerical tools in [13-15] providing the first genuine counterexample to the well-known "no-hair" conjecture; unlike other supposedly "hairy" black holes that were unstable at the end [16], see also [17-19,21-24]. Cosmological applications of the Skyrme model have also been considered [25,26].

Many of the important results in both the Skyrme model and the Einstein-Skyrme system have been derived numerically due to the highly non-linear character of the field equations and, until very recently, there were no analytic solutions with Baryon charge in these models. Analytic results are not just of academic interest. For instance, it was known that large isospin chemical potentials lead to Skyrmion instabilities on flat space. However, it is just lately that this critical behavior in the chemical potential has been fully understood thanks to an analytic formula [27-31]. Although the most relevant case corresponds to the $S U$ (3) group, many of the theoretical and numerical works have been performed for the $S U(2)$ case, which is already quite difficult in itself but not as much as the former one that not only exhibits five more generators infinitesimally, but also a non-constant curvature group manifold. However, since genuine features of the $S U$ (3) Skyrme model could have very important physical consequences it is important to pursue any hint contributing to its understanding. In the seminal works $[6,32,33]$ 
the first numerical example of a non-embedded solution was constructed. This is a genuine feature of the $S U$ (3) Skyrme model since the solution has spherical symmetry and at the same time Baryon charge equal to 2; in contrast to the $S U$ (2) Skyrme model where the only stable solution with spherical symmetry, in the hedgehog sense, has unit Baryon charge. These pioneering ideas have been generalized in [34-37]. Remarkably enough, in the present paper we provide the first analytic examples of these types. Very recently, following the techniques developed in [38-46], the first analytic self-gravitating $S U$ (2) Skyrmions have been constructed in [47]. Here it will be shown that the configurations found in [47] can be generalized to genuine self-gravitating and topologically non-trivial $S U(3)$ configurations. The resulting ansatz is based on the $S O(3)$ subgroup of $S U(3)$ that has been introduced in $[6,32,33]$. Moreover, we can define a suitable flat limit of these self-gravitating configurations in which they remain at the same time topologically non-trivial and integrable. Such a limit corresponds to confining the 4Baryon (our analytic configuration with topological charge equals to 4) in a finite volume. This construction discloses a novel finite density transition: the non-embedded solutions prevail at high Baryon density while the trivially-embedded solutions prevail at low Baryon density.

The paper is organized as follows: in the Sect. 2, the Einstein-Skyrme model is introduced. In Sect. 3, the gravitating 4-Baryon is constructed. In Sect. 4, the NUT-AdS wormhole is described. In Sect. 5, 4-Baryons living within a finite flat spatial volume are introduced. In the Sect. 6, the possibility of a phase transition arising from the competition between embedded and non-embedded solutions is discussed. Eventually, some conclusions and perspectives are presented.

\section{The Einstein-Skyrme theory}

The Einstein-Skyrme system in presence of a cosmological constant is described by the action

$$
\begin{aligned}
I[g, U]= & \int d^{4} x \sqrt{-g}\left(\frac{R-2 \Lambda}{2 \kappa}\right. \\
& \left.+\frac{K}{4} \operatorname{Tr}\left[A^{\mu} A_{\mu}+\frac{\lambda}{8} F_{\mu \nu} F^{\mu \nu}\right]\right),
\end{aligned}
$$

where $R$ is the Ricci scalar, $A_{\mu}=U^{-1} \nabla_{\mu} U$, with $U \in$ $S U(N)$ and $\nabla_{\mu}$ the covariant derivative. Moreover $F_{\mu \nu}=$ [ $\left.A_{\mu}, A_{\nu}\right], \Lambda$ is the cosmological constant, $\kappa$ the gravitational constant, and the positive couplings $K$ and $\lambda$ are fixed by experimental data. In our convention $c=\hbar=1$ and Greek indices run over the four dimensional space-time with mostly plus signature.
The complete Einstein-Skyrme field equations read

$\nabla^{\mu} A_{\mu}+\frac{\lambda}{4} \nabla^{\mu}\left[A^{\nu}, F_{\mu \nu}\right]=0, \quad G_{\mu \nu}+\Lambda g_{\mu \nu}=\kappa T_{\mu \nu}$,

where $G_{\mu \nu}$ is the Einstein tensor, and the Skyrme energymomentum tensor is defined by

$$
\begin{aligned}
T_{\mu \nu}= & -\frac{K}{2} \operatorname{Tr}\left[A_{\mu} A_{\nu}-\frac{1}{2} g_{\mu \nu} A^{\alpha} A_{\alpha}\right. \\
& \left.+\frac{\lambda}{4}\left(g^{\alpha \beta} F_{\mu \alpha} F_{\nu \beta}-\frac{1}{4} g_{\mu \nu} F_{\alpha \beta} F^{\alpha \beta}\right)\right] .
\end{aligned}
$$

The winding number for a given solution is given by

$$
B=\frac{1}{24 \pi^{2}} \int \rho_{\mathrm{B}} d^{3} x, \quad \rho_{\mathrm{B}}=\operatorname{Tr}\left[\epsilon^{i j k} A_{i} A_{j} A_{k}\right] .
$$

When the topological density $\rho_{\mathrm{B}}$ is integrated on a space-like surface, $B$ represents the Baryon number of the configuration.

\section{$3 S U(3)$ self-gravitating 4-Baryon}

Before presenting the new results, first we will shortly describe the trivial embedding into $S U(3)$ of the $S U(2)$ selfgravitating solution found in [47].

\subsection{The $S U$ (2) embedded self-gravitating Skyrmion}

The $S U(2)$ generalized hedgehog ansatz reads

$$
\begin{aligned}
U\left(x^{\mu}\right) & =Y^{0}\left(x^{\mu}\right) I \pm Y^{i}\left(x^{\mu}\right) \Lambda_{i}, \quad\left(Y^{0}\right)^{2}+Y^{i} Y_{i}=1, \\
\Lambda_{i} & =\left(\lambda_{1}, \lambda_{2}, \lambda_{3}\right), \quad Y^{0}=\cos \alpha, \quad Y^{i}=n^{i} \sin \alpha,
\end{aligned}
$$

where $n^{1}=\sin \Theta \cos \Phi, n^{2}=\sin \Theta \sin \Phi, n^{3}=\cos \Theta$ and $\left\{\lambda_{j}\right\}_{j=1, \ldots, 8}$ are the Gell-Mann matrices. Notice that $\left\{\lambda_{1}, \lambda_{2}, \lambda_{3}\right\}$ generate the $S U$ (2) subgroup of $S U(3)$. The above scalar functions are chosen in [47] as

$$
\begin{aligned}
& \Phi=\frac{\gamma+\varphi}{2}, \quad \tan \Theta=\frac{\cot \left(\frac{\theta}{2}\right)}{\cos \left(\frac{\gamma-\varphi}{2}\right)}, \\
& \tan \alpha=\frac{\sqrt{1+\tan ^{2} \Theta}}{\tan \left(\frac{\gamma-\varphi}{2}\right)},
\end{aligned}
$$

while the metric is

$d s^{2}=-d t^{2}+\rho(t)^{2}\left[(d \gamma+\cos \theta d \varphi)^{2}+d \theta^{2}+\sin ^{2} \theta d \varphi^{2}\right]$,

with the range of coordinates

$0 \leq \gamma<4 \pi, \quad 0 \leq \theta<\pi, \quad 0 \leq \varphi<2 \pi$, 
uniquely fixed by requiring the regularity of the metric. With this ansatz one can verify that the Skyrme field equations are identically satisfied, while the Einstein equations reduce to [47]

$\dot{\rho}^{2}=\frac{\Lambda}{3} \rho^{2}+\frac{\lambda \kappa K}{32 \rho^{2}}+\frac{\kappa K-2}{8}, \quad \ddot{\rho}=\frac{\Lambda}{3} \rho-\frac{\lambda \kappa K}{32 \rho^{3}}$.

Using Eqs. (4) and (8) the Baryon number of this configuration turns out to be $B=1$.

\subsection{The new $S U$ (3) non-embedded self-gravitating 4-Baryon}

To construct a self-gravitating solution with higher Baryonic charge we use the remarkable ansatz introduced in $[6,32,33]$ for a diBaryon in flat space-time. This ansatz is constructed with the subalgebra of the Gell-Mann matrices generating the subgroup $S O(3) \subseteq S U(3)$, namely $\left\{\lambda_{7},-\lambda_{5}, \lambda_{2}\right\}$. The $U$ field reads

$$
\begin{aligned}
U_{\mathrm{B}}= & \exp (i \psi) \mathbf{1}_{3 \times 3}+i \sin (\chi) \exp \left(-\frac{i \psi}{2}\right) \mathbf{T} \\
& +\left(\cos (\chi) \exp \left(-\frac{i \psi}{2}\right)-\exp (i \psi)\right) \mathbf{T}^{2}, \\
\mathbf{T}= & \vec{\Lambda} \cdot \hat{n}, \quad \hat{n}=(\sin \Theta \cos \Phi, \sin \Theta \sin \Phi, \cos \Theta), \\
\vec{\Lambda} & =\left(\lambda_{7},-\lambda_{5}, \lambda_{2}\right) .
\end{aligned}
$$

In Refs. $[6,32,33] \psi$ and $\chi$ are the radial profiles of the spherically symmetric $S U$ (3) Skyrmion, while $\Theta$ and $\Phi$ are chosen as the spherical angles. Here, we promote the four functions to new profiles depending on the coordinates of a generally curved space-time, which must be determined by solving the Skyrme field equations.

In the flat case, the Baryon charge can be different from zero even if the profile $\psi$ vanishes $[6,32,33]$ but the field equations require $\psi$ to be non-trivial. On the other hand, in the Einstein-Skyrme case one can take $\psi=0$ for the curved space-times we consider as it will be now discussed.

At this point, we need an educated ansatz for the four functions appearing in the above ansatz. A slight modification of the generalized hedgehog ansatz of [47] defined by $\psi=0$ and

$$
\begin{aligned}
\Phi & =\frac{\gamma+\varphi}{2}, \quad \tan \Theta=\frac{\cot \left(\frac{\theta}{2}\right)}{\cos \left(\frac{\gamma-\varphi}{2}\right)}, \quad \tan \left(\frac{\chi}{2}\right) \\
& =\frac{\sqrt{1+\tan ^{2} \Theta}}{\tan \left(\frac{\gamma-\varphi}{2}\right)},
\end{aligned}
$$

does the job. Indeed, the $S U$ (3) Skyrme configuration (10)(12) identically satisfies the Skyrme field equations on any metric of the form (7)-(8).
Thus, Einstein equations with the energy-momentum tensor in Eq. (3) corresponding to the Skyrme configurations defined in Eqs. (10)-( 12) reduce to

$\dot{\rho}^{2}=\frac{\Lambda}{3} \rho^{2}+\frac{\lambda \kappa K}{8 \rho^{2}}+\frac{2 \kappa K-1}{4}, \quad \ddot{\rho}=\frac{\Lambda}{3} \rho-\frac{\lambda \kappa K}{8 \rho^{3}}$.

One can write down the most general solution of Eqs. (13) following the analysis of [44]. The above Eq. (13) in the $S U$ (3) case are very similar to the $S U$ (2) one in Eq. (9), but one can notice that the coefficients appearing are different; in fact, the $S U$ (3) energy-momentum contribution is four times that of $S U(2)$. This difference also appears when computing the topological charge density $\rho_{\mathrm{B}}^{\mathrm{SU}(3)}$ of the $S U(3)$ ansatz, defined in Eqs. (10)-( 12), and the corresponding $\rho_{\mathrm{B}}^{\mathrm{SU}(2)}$ of the $S U(2)$ ansatz, defined in Eqs. (5) and (6),

$\rho_{\mathrm{B}}^{\mathrm{SU}(3)}=6 \sin \theta, \quad \rho_{\mathrm{B}}^{\mathrm{SU}(2)}=\frac{3}{2} \sin \theta$.

Taking into account Eqs. (4) and (8), the topological charge of the $S U$ (3) configuration is $B=4$. These are genuine $S U$ (3) configurations: in $S U$ (2) one cannot obtain configurations with topological charge 4 compatible with the metric in Eq. (7).

Another interesting quantity is the ratio

$\Delta=\frac{\operatorname{Vol}(S U(3))}{\operatorname{Vol}(S U(2))}=\frac{1}{2 \sqrt{2}}\left(\frac{2 K \kappa-1}{K \kappa-2}\right)^{\frac{3}{2}}$,

between the three-dimensional volume of the spatial section of the static gravitating 4-Baryons (corresponding to the static solutions of Eq. (13)) and the static gravitating Skyrmions of [47] (corresponding to the static solutions of Eq. (9)). It reveals the non-trivial strong and gravitational interactions of the system (as, in the case of four noninteracting solitons, one should expect $\Delta=4$ ).

The above regular solutions of the Einstein- $\Lambda-S U(3)$ Skyrme system defined in Eqs. (7), (8), (10), (11) and (12), are the first analytic self-gravitating Skyrmions of higher Baryonic charge in $(3+1)$-dimensions.

The fact that the Baryon charge is 4 instead of 2 as in $[6,32,33]$, is related to the compactness of the $t=$ const. hypersurfaces of the metric (7). Here, rather than requiring boundary conditions at spatial infinity as in $[6,32,33]$, one has to require periodic boundary conditions for $U_{\mathrm{B}}$ compatible with the compact spatial metric (7)-(8). This charge 4 arises due to the fact that, unlike what happens in $[6,32,33]$, the present gravitating 4-Baryons must wrap around three compact spatial directions instead of two.

\subsection{Bianchi IX}

The above construction can be further extended to a Bianchi IX cosmology. Indeed, the $S U$ (3) Skyrme field equations on 
the metric

$$
\begin{aligned}
d s^{2}= & -d t^{2}+I_{1}^{2}(\cos \theta d \gamma+d \phi)^{2} \\
& +I_{2}^{2}(\cos \phi d \theta+\sin \theta \sin \phi d \gamma)^{2} \\
& +I_{3}^{2}(\sin \phi d \theta-\sin \theta \cos \phi d \gamma)^{2},
\end{aligned}
$$

where $I_{j}=I_{j}(t)$, are still identically satisfied with the same ansatz defined in Eqs. (10), (11) and (12)! The reason behind this quite remarkable fact is that the left-invariant forms that appear in the construction of the most general Bianchi IX metric are proportional to the left-invariant forms that appear when computing the Skyrmion derivatives

$\left(U_{\mathrm{B}}\right)^{-1} \partial_{\mu} U_{\mathrm{B}}=\Omega_{\mu}^{a} \lambda_{a}$,

with the $U_{\mathrm{B}}$ defined in Eqs. (10), (11) and (12). Since the $\Omega_{\mu}^{a}$ characterizing the Skyrmionic configuration play, at the same time, also the role of "drei-bein" of the spatial metric, huge simplifications appear in the field equations. Hence, the complete set of coupled Einstein- $\Lambda$-SU (3)-Skyrme field equations (2) and (3) for the metric (14) with the ansatz in Eqs. (10), (11) and (12), reduce to a consistent dynamical system for the three Bianchi IX scale factors

$$
\begin{aligned}
& I^{(4)}-2 \mathcal{I}+4 \Lambda \mathcal{I}_{(3)}^{2}-4 \mathcal{I}_{(3)}\left(I_{1}^{\prime} I_{2}^{\prime} I_{3}+I_{1}^{\prime} I_{2} I_{3}^{\prime}+I_{1} I_{2}^{\prime} I_{3}^{\prime}\right) \\
& +\frac{K \kappa}{2}\left(\lambda I^{(2)}+4 \mathcal{I}\right)=0 \\
& \left(4 I_{1}^{4}-I^{(4)}-2 \mathcal{I}+4 I_{2}^{2} I_{3}^{2}\right) I_{2} I_{1}^{\prime} \\
& +\left(4 I_{1}^{4}-2 I^{(4)}+4\left(1-\Lambda I_{1}^{2}\right) I_{2}^{2} I_{3}^{2}\right) I_{1} I_{2}^{\prime} \\
& +4 \mathcal{I}_{(3)} I_{3}\left(I_{1} I_{1}^{\prime} I_{2}^{\prime 2}+I_{2}\left(I_{1} I_{2}\right)^{\prime} I_{1}^{\prime \prime}\right) \\
& +\frac{K \kappa}{2}\left[\lambda I_{1}\left(2 I_{1}\left(I_{1} I_{2}\right)^{\prime}-I^{(2)} I_{2}^{\prime}\right)\right. \\
& \left.+4 I_{2}\left(\mathcal{I} I_{1}^{\prime}-2 I_{2} I_{3}^{2}\left(I_{1} I_{2}\right)^{\prime}\right)\right]=0
\end{aligned}
$$

and

$$
\begin{aligned}
& \left(4 I_{2}^{4}-I^{(4)}-2 \mathcal{I}+4 I_{1}^{2} I_{3}^{2}\right) I_{1} I_{2}^{\prime} \\
& +\left(4 I_{2}^{4}-2 I^{(4)}+4\left(1-\Lambda I_{2}^{2}\right) I_{1}^{2} I_{3}^{2}\right) I_{2} I_{1}^{\prime} \\
& +4 \mathcal{I}_{(3)} I_{3}\left(I_{2} I_{2}^{\prime} I_{1}^{\prime 2}+I_{1}\left(I_{1} I_{2}\right)^{\prime} I_{2}^{\prime \prime}\right) \\
& +\frac{K \kappa}{2}\left[\lambda I_{2}\left(2 I_{2}\left(I_{1} I_{2}\right)^{\prime}-I^{(2)} I_{1}^{\prime}\right)\right. \\
& \left.+4 I_{1}\left(\mathcal{I} I_{2}^{\prime}-2 I_{1} I_{3}^{2}\left(I_{1} I_{2}\right)^{\prime}\right)\right]=0,
\end{aligned}
$$

where we have defined $I^{(2)}=I_{1}^{2}+I_{2}^{2}+I_{3}^{2}, \quad I^{(4)}=I_{1}^{4}+$ $I_{2}^{4}+I_{3}^{4}, \quad \mathcal{I}=I_{1}^{2} I_{2}^{2}+I_{1}^{2} I_{3}^{2}+I_{2}^{2} I_{3}^{2}, \quad I_{(3)}=I_{1} I_{2} I_{3}$.

As the $S U$ (3) Skyrme field equations are automatically satisfied, the above dynamical system for the scale factors $I_{j}(t)$ describes the dynamical evolution of the four self-gravitating Skyrmions. The analysis of such system can reveal many interesting features about the interplay between the gravitational and the strong interactions of these 4-Baryons. A comparison with the numerical gravitating Skyrmions constructed in [48] is useful. Their numerical solutions with Baryon charge 2 (which are based on the $S U(2)$ subgroup of $S U(3)$ ) are asymptotically flat while the present analytic solutions with Baryon charge 4 (which are based on a non-embedded ansatz) either have compact spatial sections (in the Bianchi IX sector) or possess two asymptotically NUT-AdS regions (see the section below).

\section{NUT-AdS Wormhole}

In this section we will consider the limit of vanishing Skyrme coupling $\lambda=0$. Following [47], a double-Wick rotation of the ansatz in Eq. (12) leads to $\psi=0$ and

$\Phi=\frac{t+\varphi}{2}, \quad \tan \Theta=\frac{\cot \left(\frac{\theta}{2}\right)}{\cos \left(\frac{t-\varphi}{2}\right)}$,

$\tan \left(\frac{\chi}{2}\right)=\frac{\sqrt{1+\tan ^{2} \Theta}}{\tan \left(\frac{t-\varphi}{2}\right)}$,

for the $S U$ (3) non-linear sigma model. Interestingly enough, the field equations of the $S U(3)$ non-linear sigma model with the ansatz defined in Eqs. (10), (11) and (15) on the metric

$$
\begin{gathered}
d s^{2}=\rho(\gamma)^{2}\left[-Q^{2}(d t+\cos \theta d \varphi)^{2}+d \theta^{2}+\sin ^{2} \theta d \varphi^{2}\right] \\
+d \gamma^{2},
\end{gathered}
$$

are identically satisfied. A direct computation shows that the coupled Einstein- $\Lambda$-SU (3)-non-linear sigma model field equations (2) and (3) with $\lambda=0$ are satisfied for

$\rho(\gamma)=\sqrt{\frac{3(\kappa K-2)}{4|\Lambda|}} \cosh \left(\sqrt{\frac{|\Lambda|}{3} \gamma}\right), \quad Q^{2}=\kappa K$,

provided $\Lambda<0$. Notice that the square of the NUT parameter $Q^{2}$ is four times the result for $S U$ (2) [47]. The above metric represents a traversable Lorentzian wormhole with NUT-AdS asymptotic supported by a reasonable physical source (see [47,49] ); the non-linear sigma models do not violate energy conditions. The well-known no-go results on the existence of wormholes are avoided due to the presence of a NUT parameter. The $S U$ (3) wormhole throat is larger than the one of the $S U(2)$ configuration in $[47,49]$ since the present matter field possesses a higher topological charge.

\section{4-Baryons at finite density}

The first analytic solutions with non-vanishing Baryon charge in the $S U$ (2) Skyrme model on flat space have been 
found by adapting the self-gravitating solution found in [47] to a flat space with finite volume in [27]. A similar construction also works in the present case. The sector explored below describes four low-energy Baryons confined within a finite volume in flat space.

Consider the $S U$ (3) Skyrme configuration defined by $\psi=$ 0 and

$$
\begin{aligned}
& \Phi=\frac{\gamma+\varphi}{2}, \quad \tan \Theta=\frac{\tan H(t, z)}{\cos \left(\frac{\gamma-\varphi}{2}\right)}, \\
& \tan \left(\frac{\chi}{2}\right)=\frac{\sqrt{1+\tan ^{2} \Theta}}{\tan \left(\frac{\gamma-\varphi}{2}\right)},
\end{aligned}
$$

together with Eqs. (10) and (11) in a flat metric of the form

$d s^{2}=-d t^{2}+L_{0}^{2}\left[d z^{2}+d \gamma^{2}+d \varphi^{2}\right]$,

where $L_{0}$ has dimension of length and represents the size of the box in which the Baryons are confined, and the dimensionless coordinates have the following ranges

$0 \leq z \leq 2 \pi, \quad 0 \leq \gamma \leq 4 \pi, \quad 0 \leq \varphi \leq 2 \pi$.

The topological density is again different from the $S U(2)$ case since

$\rho_{\mathrm{B}}^{\mathrm{SU}(3)}=-12 \sin (2 H) \partial_{z} H, \quad \rho_{\mathrm{B}}^{\mathrm{SU}(2)}=-3 \sin (2 H) \partial_{z} H$.

Considering the boundary conditions as $H(t, 0)=0$ and $H(t, 2 \pi)= \pm \frac{\pi}{2}$, the $S U$ (3) topological charge is \pm 4 (which is four times the charge found in [27-29]). Concretely, these Skyrmions confined to a finite volume can only have charges $-4,0$, or 4 (while in the $S U$ (2) case defined in [27-29], the corresponding Skyrmions can only have charges $-1,0$, or 1 ). This confirms the genuine $S U$ (3) nature of the ansatz defined in Eqs. (10), (11) and (17).

The $S U$ (3) Skyrme field equations on the flat metric (18) for the Baryon-like ansatz (10), (11) and (17) become a single partial differential equation for a scalar a profile

$\square H-\frac{\lambda}{8 L_{0}^{2}\left(2 L_{0}^{2}+\lambda\right)} \sin (4 H)=0$.

Hence, the eight coupled $S U$ (3) Skyrme field equations collapse to a single integrable PDE. This opens the intriguing possibility to analyze many interesting non-trivial properties of these multi-Skyrmions confined to a finite volume using Sine-Gordon theory such as possible phase transitions between the trivial embedding of the $S U$ (2) solutions [27] and the present non-embedded $S U$ (3) solutions living in the same box.

At this point it is important to recall that the value 4 of the Baryonic charge cames only from the nature of the $S U$ (3) ansatz and the compactness of the space-time under consideration, both, in the present case and in the previous gravitating configurations. Using the same boundary conditions for the usual $S U$ (2) Skyrme field is not possible to obtain $B=4$. In fact, Eqs. (17) and (19) describes a bound state of 4-Baryons whose energy density is localized. In the next section will be show that indeed, the energy of the 4-Baryon state is different from the one of the four Skyrmions on the $S U(2)$ ansatz.

\section{A novel transition at finite Baryon density}

In the present section we will show that, within the same box of finite volume, there is a competition between embedded and non-embedded configurations with the same Baryonic charge, the control parameter of the transition being the Baryon density. We show here below that at high Baryon density non-embedded solutions are favoured over embedded solutions (and vice-versa at low Baryon density).

A suitable ansatz to describe $S U$ (3) configurations living in the flat box defined above that are trivial embedding of $S U(2)$ into $S U(3)$ is

$$
\begin{aligned}
\vec{\Lambda} & =\left(\lambda_{1}, \lambda_{2}, \lambda_{3}\right), \quad \Phi=\frac{p \gamma+q \varphi}{2}, \\
\tan \Theta & =\frac{\tan H(t, z)}{\cos \left(\frac{p \gamma-q \varphi}{2}\right)}, \quad \tan (\chi)=\frac{\sqrt{1+\tan ^{2} \Theta}}{\tan \left(\frac{p \gamma-q \varphi}{2}\right)},
\end{aligned}
$$

where $p$ and $q$ are non-vanishing integer numbers (see [50]) and $\lambda_{1}, \lambda_{2}, \lambda_{3}$ the first three Gell-Mann matrices.

As we explained in Section V, as well as in [50], both in the 4-Baryon case (Eqs. (10) and (17)) and in the case of trivially embedded solutions (Eqs. (10) and (20)) the consistent boundary conditions for the profile $H(t, r)$ are

$H(t, 0)=0, \quad H(t, 2 \pi)= \pm \frac{\pi}{2}$.

The topological densities read

$\rho_{\mathrm{B}}^{\mathrm{SU}(3)}=-12 \sin (2 H) \partial_{z} H$,
$\rho_{\mathrm{B}}^{\mathrm{SU}(2)}=-3 p q \sin (2 H) \partial_{z} H$.

We know that the topological charge in the $S U(3)$ case is $B^{\mathrm{SU}(3)}=4$, while the topological charge in the trivially embedded solutions with the above slightly generalized ansatz is

$B=p q$,

where the integers $p$ and $q$ are the ones appearing in the ansatz in Eqs. (10) and (20) (see also [50]).

Thus, if we choose

$p q=4$, 
then we will have two inequivalent configurations ${ }^{1}$ (the one in Eqs. (10) and (17) as well as the one in Eqs. (10) and (20)) with the same charge living in the same box. The natural question is:

Which type of configuration is energetically favoured?

Thanks to the remarkable properties of the ansatz described above, one can answer this question explicitly. The first step is to notice that in both cases (the one in Eqs. (10) and (17) as well as the one in Eqs. (10) and (20)) the $S U$ (3) Skyrme field equations in the flat metric (18) reduce to a single partial differential equation of the form

$\square H-\beta \sin (4 H)=0$,

with

$\beta_{\mathrm{SU}(3)}=\frac{\lambda}{8 L_{0}^{2}\left(2 L_{0}^{2}+\lambda\right)}$,

$\beta_{\mathrm{SU}(2)}=\frac{p^{2} q^{2} \lambda}{4 L_{0}^{2}\left(4 L_{0}^{2}+\lambda\left(p^{2}+q^{2}\right)\right)}$,

for (17) and (20), respectively.

For static configurations $H(t, r)=H(r)$, Eq. (21) admits a first integral

$\left(H^{\prime}\right)^{2}+\frac{1}{2} \beta \cos (4 H)=I_{0}$,

with the integration constant defined using the boundary conditions as

$$
\int_{0}^{2 \pi} d r=\int_{0}^{\frac{\pi}{2}} \frac{d H}{\sqrt{I_{0}-\frac{1}{2} \beta \cos (4 H)}}=2 \pi .
$$

The energy density for these configurations are given by

$$
\begin{aligned}
T_{00}^{\mathrm{SU}(3)}= & -\frac{K}{16 L_{0}^{4}}\left(16 L_{0}^{2}+\lambda(1-\cos (4 H))+\frac{16}{L^{2}}\left(2 L_{0}^{2}+\lambda\right) H^{\prime 2}\right), \\
T_{00}^{\mathrm{SU}(2)}= & -\frac{K}{16 L_{0}^{4}}\left(8 L_{0}^{2}\left(p^{2}+q^{2}\right)+p^{2} q^{2} \lambda(1-\cos (4 H))\right. \\
& \left.+8\left(4 L_{0}^{2}+\left(p^{2}+q^{2}\right) \lambda\right) H^{\prime 2}\right) .
\end{aligned}
$$

TABLE $1^{2}$ shows the energy comparison between the $S U$ (2) and the $S U$ (3) configurations with Baryon charge $B=4$, while it varies the size of the box (measured by $L_{0}$ ). We have fixed $K=2, \lambda=1$ (which means that we are measuring length in $\mathrm{fm}$ ). One can see that a phase transition

\footnotetext{
1 Using the elegant arguments in [6], one can easily see that these two configurations cannot be deformed into each other using global isospin transformations.

2 The notation $E_{X, Y}^{S U(2)}$ means "the energy of the $S U(2)$ configuration in Eqs. (10) and (20) with $p=X$ and $q=Y$ ". In the table $X Y=4$ as we need to compare configurations with the same topological charge.
}

Table 1 Phase transition $S U(3)$ vs. $S U(2) \subset S U(3)$ at finite density

\begin{tabular}{lllll}
\hline$L_{0}$ & $E_{2,2}^{S U(2)}$ & $E_{1,4}^{S U(2)}$ & $E_{4,1}^{S U(2)}$ & $E^{S U(3)}$ \\
\hline 0.01 & 283.131 & 416.22 & 416.22 & 147.81 \\
0.1 & 29.627 & 44.317 & 44.317 & 16.198 \\
0.5 & 12.288 & 21.925 & 21.925 & 10.082 \\
1 & 16.040 & 31.359 & 31.359 & 15.675 \\
1.4 & 20.455 & 41.033 & 41.033 & 20.899 \\
2 & 27.637 & 56.414 & 56.414 & 29.054 \\
3 & 40.093 & 82.814 & 82.814 & 42.933 \\
5 & 65.557 & 136.421 & 136.421 & 70.999 \\
10 & 129.980 & 271.440 & 271.440 & 141.528 \\
\hline
\end{tabular}

appears at ${ }^{3} L_{0}^{*} \approx 1.4$. When $L_{0}<L_{0}^{*}$ then the 4-Baryon are favoured over the trivially embedded solutions (and viceversa when $L_{0}>L_{0}^{*}$ ). The appearance of this transition is related to the fact that 4-Baryons deal more efficiently with the well known repulsive interaction between Skyrmions. From the plots of the energy densities below for the two types of configurations one can see that, at high Baryon density, it pays off that the 4-Baryon is "less peaked" around its maximum while at low Baryon densities the energy densities of both types of solutions become relatively flat and, in such cases, the trivially embedded solutions prevail.

Fig. 1 shows the energy density of the three relevant configurations, for three different values of the size of the box.

At last, we make a small observation about the quantization of the solutions. Since, with the above ansatz, the hedgehog property holds (as the 8 coupled $S U(3)$ field equations reduce to a single PDE for the profile $H$ ). The small fluctuations of the profile $H$ around these solutions are described by the effective action obtained replacing the ansatz itself into the original $S U$ (3) Skyrme action ( $[51,52])$. Thus, the quantization of the 4-Baryon living in the finite box defined above can be analyzed using known results on the sine-Gordon theory plus the semiclassical quantization of the Isospin degrees of freedom described in $[32,33]$. This observation also shows that the flat configurations constructed in the manuscript are stable, at least, under the perturbations which keep the hedgehog properties. Namely, the flat solutions considered here are stable under the following type of perturbations (see the analysis in [51] and [52]):

$H(t, r) \rightarrow H(t, r)+\varepsilon u(t, r), 0<\varepsilon \ll 1$.

We hope to come back on the interesting but rather difficult problem of the full stability analysis (which must be analyzed numerically) in a future publication.

\footnotetext{
3 It is worth to mention that at this scale, the Skyrme model is still well within its range of validity.
} 

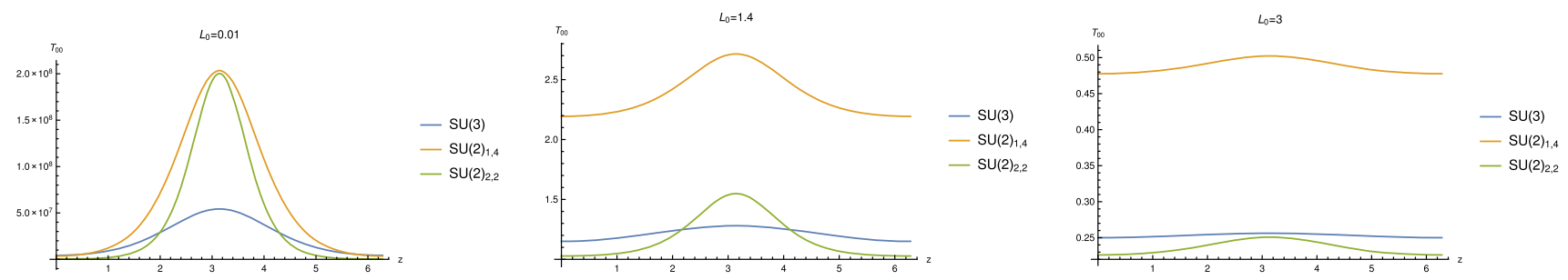

Fig. 1 Energy densities for different values of $L_{0}$. We can see that at high Baryon density it is convenient that the 4-Baryon is "less localized" around its maximum, and at low Baryon density the energy density for both types of solutions tend to flatten

\section{Conclusions and perspectives}

The first regular analytic self-gravitating Skyrmions of higher Baryon charge have been constructed. The space-time corresponds to a general Bianchi IX cosmology whose three scales factors evolve accordingly, while the Skyrme field equations are identically satisfied in the sector with Baryon charge 4 . All these solutions disclose genuine features of the $S U$ (3) Skyrme model. Traversable wormholes with NUTAdS asymptotic supported by an $S U$ (3) regular solitonic solution of the resulting non-linear sigma model are also constructed. Also, a suitable flat limit in a finite volume of the self-gravitating Skyrme configurations can also be defined. In this case, the eight $S U$ (3) Skyrme field equations become the Sine-Gordon field theory without sacrificing the higher Baryon charge. This flat limit explicitly describes charge 4 Baryons confined in a box. The present formalism discloses the existence of a novel transition between embedded and non-embedded configurations with the same Baryonic charge. The control parameter of this transition is the Baryon density: at high Baryon densities, it is energetically convenient to have non-embedded solutions while, at low Baryon densities, trivially embedded solutions prevail. To the best of authors knowledge, this phenomenon related to the competition between embedded and non-embedded solutions at finite Baryon density is new. The reason is that the present techniques are especially suitable to deal with Skyrmions and 4-Baryons at finite density as these techniques allow to determine how relevant physical properties of these solitons depend on the Baryon density. Without these informations it would have been impossible to disclose such possibility.

Acknowledgements M.L. and A.V. appreciate the support of FONDECYT postdoctoral grants 3190873 and 3200884 . This work has been partially funded by the Fondecyt Grants 1200022, 1181047, and the Conacyt Grant A1-S-11548. The Centro de Estudios Científicos (CECs) is funded by the Chilean Government through the Centers of Excellence Base Financing Program of Conicyt. We dedicate this work to Murray Gell-Mann, an eight-leagues giant of physics.

Data Availability Statement This manuscript has no associated data or the data will not be deposited. [Authors' comment: This manuscript has no associated data since it is mainly a theoretical work.]
Open Access This article is licensed under a Creative Commons Attribution 4.0 International License, which permits use, sharing, adaptation, distribution and reproduction in any medium or format, as long as you give appropriate credit to the original author(s) and the source, provide a link to the Creative Commons licence, and indicate if changes were made. The images or other third party material in this article are included in the article's Creative Commons licence, unless indicated otherwise in a credit line to the material. If material is not included in the article's Creative Commons licence and your intended use is not permitted by statutory regulation or exceeds the permitted use, you will need to obtain permission directly from the copyright holder. To view a copy of this licence, visit http://creativecomm ons.org/licenses/by/4.0/.

Funded by SCOAP . $^{3}$.

\section{References}

1. E. Witten, Nucl. Phys. B 223, 422 (1983)

2. E. Witten, Nucl. Phys. B 223, 433 (1983)

3. T.H.R. Skyrme, Proc. R. Soc. Lond. A 260, 127 (1961)

4. T.H.R. Skyrme, Proc. R. Soc. Lond. A 262, 237 (1961)

5. T.H.R. Skyrme, Nucl. Phys. 31, 556 (1962)

6. A.P. Balachandran, V.P. Nair, N. Panchapakesan, S.G. Rajeev, Phys. Rev. D 28, 2830 (1983)

7. D. Finkelstein, J. Rubinstein, J. Math. Phys. 9, 1762 (1968)

8. A.P. Balachandran, H. Gomm, R.D. Sorkin, Nucl. Phys. B 281, 573 (1987)

9. G.S. Adkins, C.R. Nappi, E. Witten, Nucl. Phys. B 228, 552 (1983)

10. N. Manton, P. Sutcliffe, Topological Solitons (Cambridge University Press, Cambridge, 2007)

11. D. Auckly, M. Speight, Commun. Math. Phys. 263, 173 (2006)

12. S. Krusch, M. Speight, Commun. Math. Phys. 264, 391 (2006)

13. H. Luckock, I. Moss, Phys. Lett. B 176, 341 (1986)

14. S. Droz, M. Heusler, N. Straumann, Phys. Lett. B 268, 371 (1991)

15. S. Droz, M. Heusler, N. Straumann, Phys. Lett. B 271, 61 (1991)

16. P. Bizon, Acta Phys. Polon. B 25, 877 (1994). [gr-qc/9402016]

17. N.K. Glendenning, T. Kodama, F.R. Klinkhamer, Phys. Rev. D 38, 3226 (1988)

18. B.M.A.G. Piette, G.I. Probert, Phys. Rev. D 75, 125023 (2007)

19. G.W. Gibbons, C.M. Warnick, W.W. Wong, J. Math. Phys. 52, 012905 (2011)

20. S. Nelmes, B.M. Piette, Phys. Rev. D 84, 085017 (2011)

21. P. Bizon, T. Chmaj, Phys. Rev. D 58, 041501 (1998)

22. P. Bizon, T. Chmaj, A. Rostworowski, Phys. Rev. D 75, 121702 (2007)

23. S. Zajac, Acta Phys. Polon. B 40, 1617 (2009)

24. S. Zajac, Acta Phys. Polon. B 42, 249 (2011)

25. F. Canfora, A. Giacomini, S.A. Pavluchenko, Phys. Rev. D 90, 043516 (2014)

26. L. Parisi, N. Radicella, G. Vilasi, Phys. Rev. D 91, 063533 (2015) 
27. P. Alvarez, F. Canfora, N. Dimakis, A. Paliathanasis, Phys. Lett. B 773, 401 (2017)

28. L. Aviles, F. Canfora, N. Dimakis, D. Hidalgo, Phys. Rev. D 96, 125005 (2017)

29. F. Canfora, M. Lagos, S.H. Oh, J. Oliva, A. Vera, Phys. Rev. D 98(8), 085003 (2018)

30. F. Canfora, Eur. Phys. J. C 78(11), 929 (2018)

31. F. Canfora, S.-H. Oh, A. Vera, Eur. Phys. J. C 79(6), 485 (2019)

32. A.P. Balachandran, A. Barducci, F. Lizzi, V.G.J. Rodgers, A. Stern, Phys. Rev. Lett. 52, 887 (1984)

33. A.P. Balachandran, F. Lizzi, V.G.J. Rodgers, A. Stern, Nucl. Phys. B 256, 525 (1985)

34. V.B. Kopeliovich, B.E. Schwesinger, B.E. Stern, JETP Lett. 62, 185 (1995)

35. A. Din, W.J. Zakrzewski, Nucl. Phys. B 174, 397 (1980)

36. T. Ioannidou, B. Piette, W.J. Zakrzewski, J. Math. Phys. 40, 6223 (1999)

37. Y. Brihaye, B. Hartmann, T. Ioannidou, W. Zakrzewski, Phys. Rev. D 69, 124035 (2004)

38. F. Canfora, H. Maeda, Phys. Rev. D 87, 084049 (2013)

39. F. Canfora, Phys. Rev. D 88, 065028 (2013)

40. F. Canfora, F. Correa, J. Zanelli, Phys. Rev. D 90, 085002 (2014)
41. F. Canfora, M. Kurkov, M. Di Mauro, A. Naddeo, Eur. Phys. J. C 75, 443 (2015)

42. S. Chen, Y. Li, Y. Yang, Phys. Rev. D 89, 025007 (2014)

43. L.A. Ferreira, JHEP 1707, 039 (2017)

44. F. Canfora, A. Paliathanasis, T. Taves, J. Zanelli, Phys. Rev. D 95, 065032 (2017)

45. A. Giacomini, M. Lagos, J. Oliva, A. Vera, Phys. Lett. B 783, 193 (2018)

46. M. Astorino, F. Canfora, M. Lagos, A. Vera, Phys. Rev. D 97, 124032 (2018)

47. E. Ayon-Beato, F. Canfora, J. Zanelli, Phys. Lett. B 752, 201 (2016)

48. H. Sato, N. Sawado, Phys. Lett. B 660, 72-79 (2008)

49. F. Canfora, N. Dimakis, A. Paliathanasis, Phys. Rev. D 96, 025021 (2017)

50. F. Canfora, N. Dimakis, A. Paliathanasis, Eur. Phys. J. C 79(2), 139 (2019)

51. S. Coleman, Aspects of Symmetry, Erice Lectures (Cambridge University Press, Cambridge, 1985)

52. M. Shifman, Advanced Topics in Quantum Field Theory: A Lecture Course (Cambridge University Press, Cambridge, 2012) 